\title{
Epithelial morphological response to soft hydrogel contact lenses
}

\author{
J P G BERgManson, C MONTAgue RUBEN, ANd L W-F CHU \\ From the Institute for Contact Lens Research, University of Houston, Texas, USA
}

SUMMARY Hydrogel (HEMA) contact lenses of increased thickness were worn by owl monkeys (Aotus tivergatus) under open and closed eye conditions to study ultrastructurally the corneal epithelial response to increased stress. The $0.07 \mathrm{~mm}$ thick lens with a calculated $\mathrm{Dk} / \mathrm{L}$ of $13 \mathrm{had}$ minimal effect on the epithelium, while thicker $(0 \cdot 2-0 \cdot 4 \mathrm{~mm})$ lenses with a Dk/L of $4 \cdot 2-2 \cdot 2$ caused an epithelial thinning without oedema. This thinning was due to a loss of superficial cells and a flattening of the remaining ones. Factors in the mechanism of epithelial thinning without oedema may include anoxia, trauma, lid pressure, and lens weight. The $0.4 \mathrm{~mm}$ thick lens offers negligible gas transmission in the closed eye, and after 48 hours much of the epithelium was stripped, while oedema and degenerative cytoplasmic changes were prominent in areas of surviving cells. In such areas the thickness of the epithelium was reduced to one or two layers of cells. The basement membrane was in all experiments unharmed by hydrogel contact lens wear. The epithelial innervation was maintained in all corneas except those of the closed eye, and this finding correlates well with the good corneal touch threshold in soft contact lens wearers.

Although the hydrogel contact lenses have been commercially available for over a decade, reports on primate controlled studies of the ultrastructural response to such lenses are sparse. ${ }^{1}$ There is an abundance of physiological and pathological evidence of individual variations in response as seen in the human at clinical levels of observations. ${ }^{2}$

The present study was designed to examine the corneal epithelial stress effects of different thicknesses of soft lenses over various time periods and to study this on the primate eye by means of the electron microscope. Furthermore, the contact lens oxygen gas flow was measured by calculation and the lens pressure effects correlated with degrees of anoxia.

The results will be compared with the recent study in the same laboratory of Bergmanson and $\mathrm{Chu}^{3}$ They studied primates wearing hard non-permeable corneal lenses (PMMA) for 2 to 24 hours, and their results indicated epithelial loss, atrophy and oedema.

\section{Material and methods}

Hydrogel lenses of different thicknesses (Table 1) were fitted on four owl monkeys (Aotus tivergatus),

Correspondence to Professor C M Ruben, Institute for Contact Lens Research, University of Houston, University Park, Houston, Texas 77004, USA. while three additional owl monkeys served as control. By varying the thickness and the wearing time of the hydrogel lenses different levels of epithelial stress were achieved (Table 2). The monkeys wore similar lenses in both eyes except for the closed-eye part of the experiment, when one eye was left open and without a lens (Table 1). The contact lenses were calculated to have $\mathrm{Dk} / \mathrm{L}$ values according to the formulations described by Fatt and St Helen. ${ }^{4}$ This value is based on the material permeability (Dk) to oxygen at normal atmospheric pressure and to the thickness (L) (Table 2).

Table 1 Experimental protocol

\begin{tabular}{|c|c|c|c|c|}
\hline \multicolumn{2}{|c|}{ Monkey } & Lens thickness & Wearing time (hours) & Lid position \\
\hline 1 & OD & 0.07 & 2 & Open \\
\hline & OS & 0.07 & 8 & Open \\
\hline 2 & OD & $0 \cdot 2$ & 15 & Open \\
\hline & OS & $0 \cdot 2$ & 15 & Open \\
\hline 3 & OD & $0 \cdot 4$ & 12 & Open \\
\hline & OS & 0.4 & 12 & Open \\
\hline 4 & OD & 0.4 & 48 & Closed \\
\hline & OS & No lens & & Open \\
\hline 5 & $\begin{array}{l}\text { OD } \\
\text { OS }\end{array}$ & Nolens Contr & N/A & Open \\
\hline 6 & $\begin{array}{l}\text { OD } \\
\text { OS }\end{array}$ & Nolens Contr & N/A & $\begin{array}{l}\text { Open } \\
\text { Open }\end{array}$ \\
\hline 7 & OD & Nolens Contr & N/A & Open \\
\hline
\end{tabular}


Table 2 Specification of hydrogel lenses used in the experiment

\begin{tabular}{|c|c|c|c|c|c|c|c|}
\hline & Material & $\begin{array}{l}\mathrm{H}_{2} \mathrm{O} \\
\text { content }\end{array}$ & $\begin{array}{l}\text { Thick } \\
\operatorname{mm}(t) *\end{array}$ & $\begin{array}{l}\text { Overall } \\
\text { Diam. }(O D) \dagger\end{array}$ & $\begin{array}{l}\text { Base } \\
\text { curve }\end{array}$ & Power & $D k / L \ddagger$ \\
\hline Thin lenses & HEMA & $38 \%$ & 0.07 & $14 \cdot 6$ & $8 \cdot 50$ & $-3 \cdot 00$ & 13 \\
\hline Medium thick lenses & HEMA & $38 \%$ & $0 \cdot 20$ & $14 \cdot 5$ & $\begin{array}{l}8 \cdot 15 \\
8 \cdot 30\end{array}$ & Plano to -0.50 & $4 \cdot 5$ \\
\hline Thick lenses & HEMA & $38 \%$ & 0.40 & $14 \cdot 0$ & $\begin{array}{l}8 \cdot 15 \\
8 \cdot 30\end{array}$ & Plano to -0.50 & $2 \cdot 2$ \\
\hline
\end{tabular}

Tolerances: ${ }^{*} \mathrm{t}= \pm 0 \cdot 02 . \dagger \mathrm{OD}= \pm 0 \cdot 50$. $\ddagger$ Fatt and St Helen. ${ }^{4}$

The keratometry measurements of these animals ranged between $r=7.49 \mathrm{~mm}(45.5 \mathrm{D})$ and $\mathrm{r}=6.59 \mathrm{~mm}$ $(51.25 \mathrm{D})$, and the mean corneal diameter was 13.0 $\mathrm{mm} \pm 0.5 \mathrm{~mm}$ (SD). The contact lens fittings were not tight or centrally steep, allowing a lens movement of about $1 \mathrm{~mm}$ on pressure, and in all cases limbal coverage was present. This fit was therefore consistent with acceptable human clinical criteria.

No sedation or topical anaesthetic was used during the experiment except in the single instance of the animal wearing a lens for 48 hours. In the last case the animal was anaesthetised with pentobarbitone (Nembutal) only for the lens insertion and surgical suturing of the lids. The closed eye was effected by cutting the inner half of the lid margin with a razor knife and using a continuous 4 'o's silk suture along the whole width of the lids completely to close the palpebral aperture. All animals were kept in an alert state while wearing the lenses.
At the end of the experimental period the animals were deeply anaesthetised and the eyes enucleated; they were then killed by a fatal dose of the anaesthetic. The enucleated eyes were immediately placed in fixative (3\% glutaraldehyde in $0.2 \mathrm{M}$ cacodylate buffer at $\mathrm{pH} 7 \cdot 2$ ). The central half of the cornea was dissected free and divided into sectors with the apex towards the centre. Sectors from different orientations were selected for light and electron microscope observations. The tissue thus examined amounted to approximately a quarter of the cornea.

After being poststained in $1 \%$ osmium tetroxide for 3 hours the tissues were dehydrated through an alcohol series and embedded in Epon 812. Semithin $(1 \mu \mathrm{m})$ light microscope sections were stained in toluidine blue, while the thin sections for the electron microscope were stained firstly in $3.5 \%$ uranyl acetate and subsequently in Reynold's lead citrate. The ultrastructural observations were made with a Jeol 100C electron microscope.
Fig. 1 Normal epithelium of noncontact lens wearing eye. The epithelium is formed by approximately five layers of tightly packed cells. The internal basal cell (B) layer adheres to a basement membrane. A squamous cell (S) shows separation of the nuclear membranes. (Control owl monkey, $\times 4380$ ).

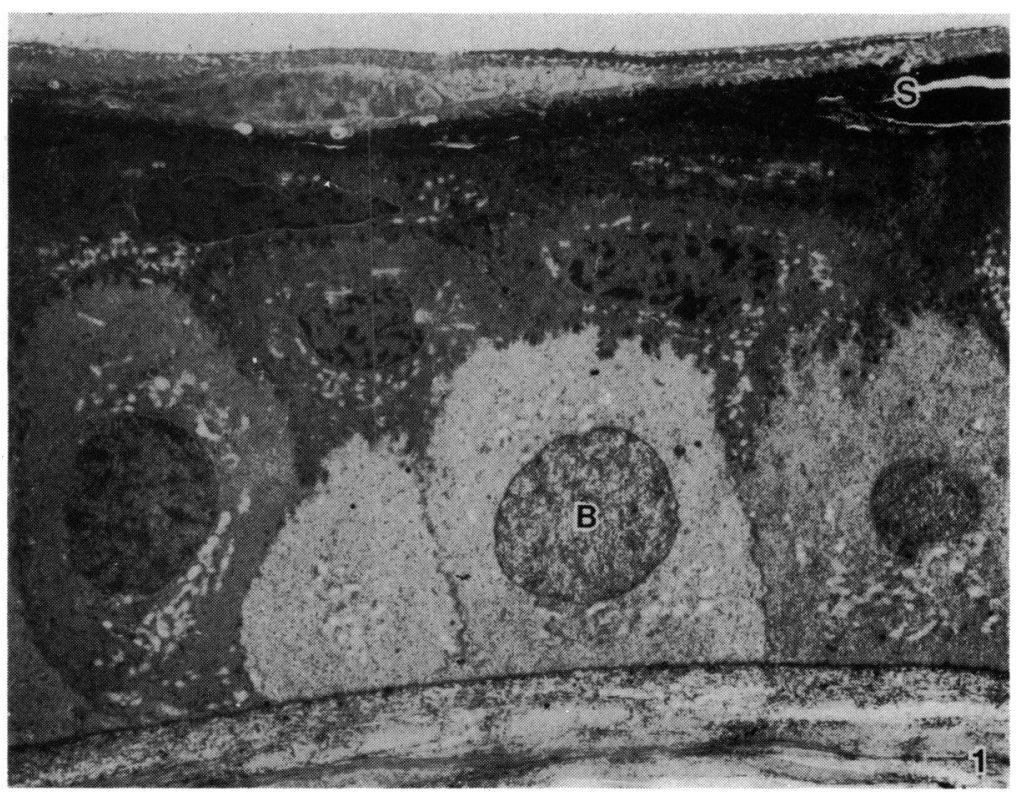




\section{Results}

CONTROL EYE

Five to six layers of cells formed the epithelium. The tightly packed cells adhered to each other through numerous desmosomes, while internally the columnar basal cells were anchored by hemidesmosomes to the basement membrane, which in tranverse section was linear (Fig. 1). Squamous cells occasionally displayed cytoplasmic pallor, separation of the nuclear membranes, and partial loss of contact to adjacent cells.

MINIMAL EPITHELIAL STRESS (THIN HYDROGEL LENSES IN OPEN EYE)

One monkey wore $0.07 \mathrm{~mm}$ thick lenses for two and eight hours. The corneal epithelium of the eye that had worn the lens for two hours showed no morphological variation from the control. Eight hours of thin lens wear caused minimal epithelial changes, which were restricted to a small increase in atrophic cells along the surface (Fig. 2). All other cells had maintained a normal shape and cytoplasm. No spaces between cells were observed.

MODERATE EPITHELIAL STRESS (MEDIUM TO THICK HYDROGEL LENSES IN OPEN EYE)

One animal wore $0.2 \mathrm{~mm}$ thick lenses, while another wore $0.4 \mathrm{~mm}$ thick lenses. The wearing times were 15 and 12 hours respectively. All eyes showed corneal epithelial thinning, which was only marginally more advanced in the monkey wearing the $0.4 \mathrm{~mm}$ thick HEMA lens. In most places the epithelium was reduced to three to four cells thickness (Fig. 3). In addition to the loss of superficial cells flattening of the remaining cells accounted for part of the overall reduction in epithelial thickness (Figs. 3, 4). The surface squamous cells had generally lost their normal cytoplasmic texture. There were no further degenerative changes in the other remaining cells other than the indicated cell deformation as well as irregularities of some nuclei. The cells remained

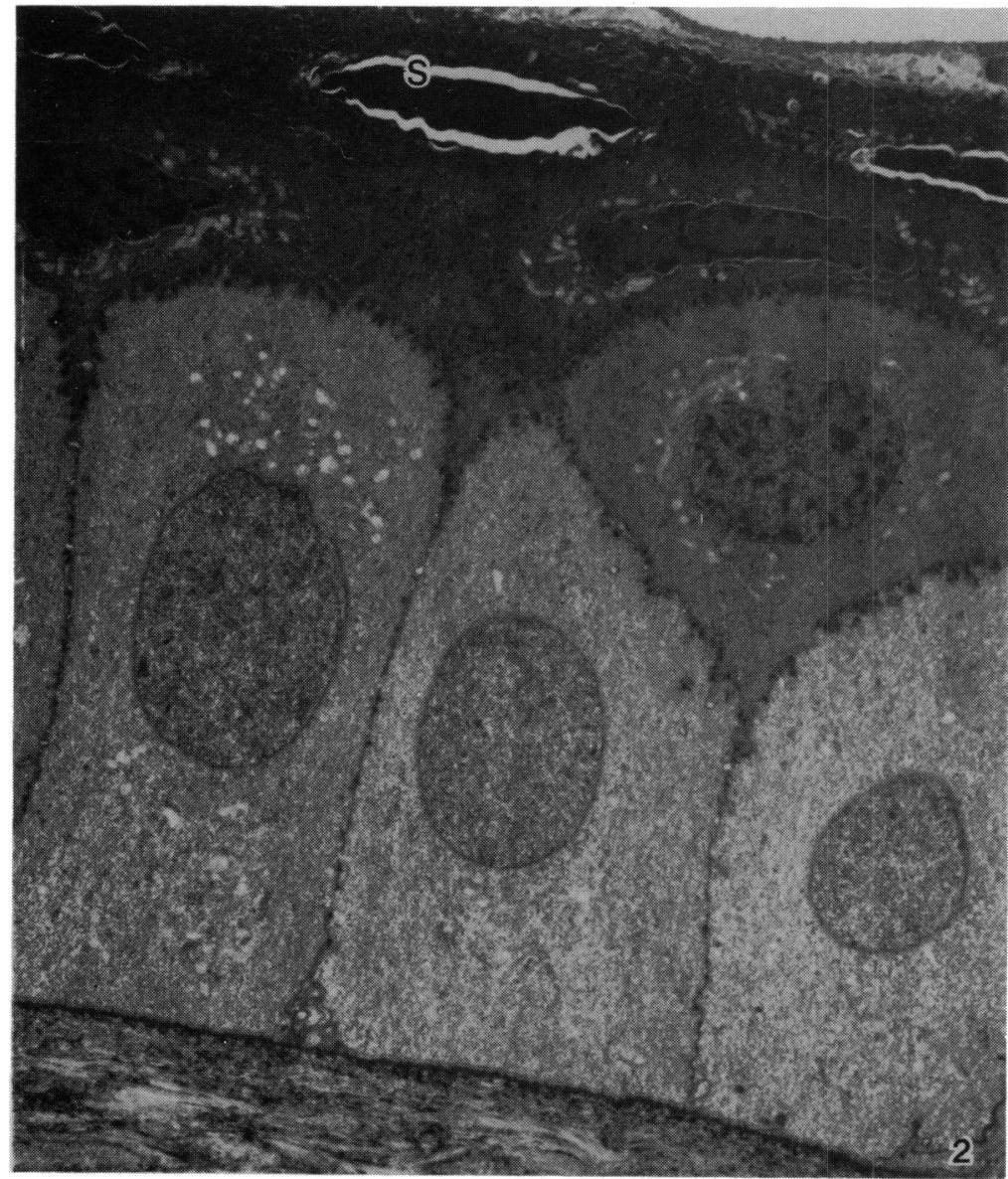

Fig. 2 Thin contact lens wearing eye. The surface layer of cells have lost their normal texture. The nuclear membranes have separated in one squamous cell (S). (Owl monkey wearing of $0.07 \mathrm{~mm}$ thick HEMA lens for 8 hours, open eye, $\times$ 4000). 
Fig. 3 Medium thick contact lens wearing eye with thinned epithelium. The epithelium is reduced to three or four cells thickness. Flattening of especially the basal cell (B) layer has further reduced the overall thickness. The cytoplasm appears normal and the cells are tightly packed. $(\mathrm{Owl}$ monkey wearing $0.2 \mathrm{~mm}$ thick HEMA lens for 15 hours, open eye, $\times$ 4255).

Fig. 4 Medium thick contact lens wearing eye with transitional zone of epithelial thinning. The surface cells (A) have lost their normal texture while the deeper cells show a graduated flattening of the five layers of tightly packed cells. The internal basal cell (B) layer adheres to a basement membrane. A squamous cell (S) shows separation of nuclear membranes. (Owl monkey wearing $0.2 \mathrm{~mm}$ thick HEMA lens for 15 hours, open eye, $\times 3830)$.

Fig. 5 Thick contact lens wearing eye normal epithelial nerve terminal. An axon (arrowhead) in the internal aspect of the basal cell (B) layer terminates in a swelling $(0.7 \mu \mathrm{m})$ (triangle) filled with mitochondria. (Owl monkey wearing a $0.4 \mathrm{~mm}$ thick HEMA lens for 12 hours, open eye, $\times 25530$ ).
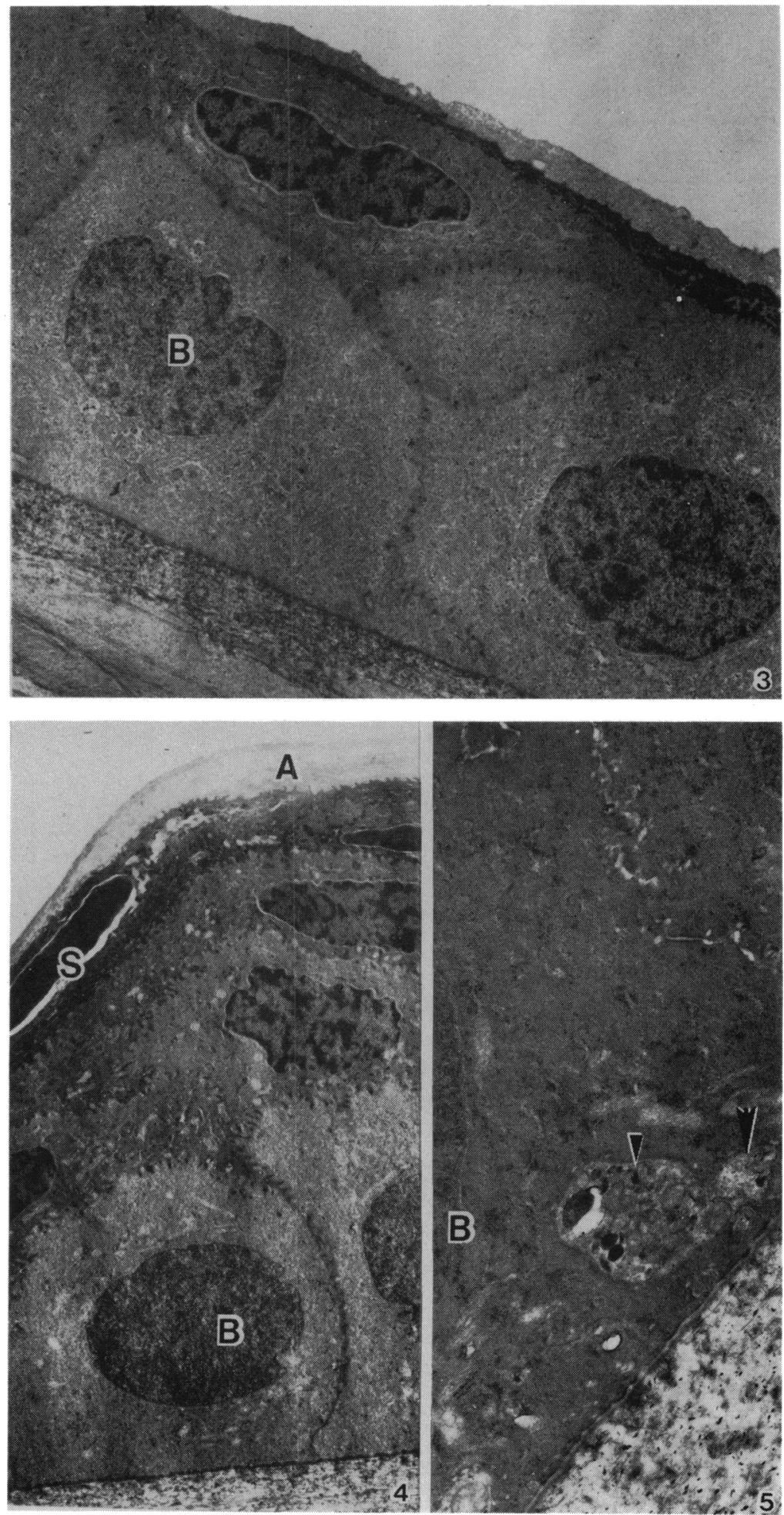


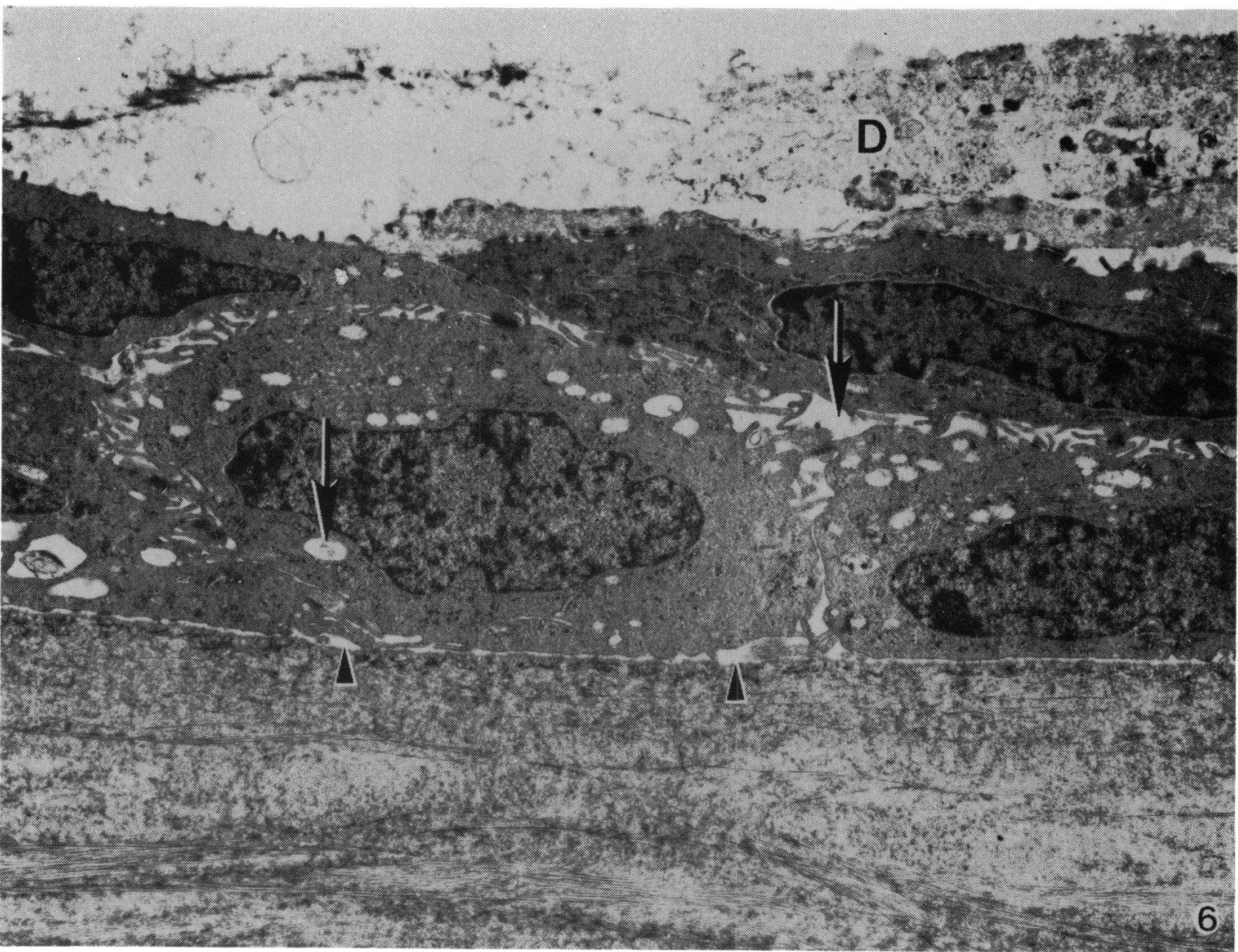

Fig. 6 Thick contact lens wearing eye with thinned oedematous epithelium. The epithelium is reduced to two layers of cells with some debris (D) along the surface. All cells and nuclei show marked deformation. Oedema (arrows) is evident intra-and intercellularly. Separations (triangles) have appeared between the basal cells and the basement membrane. (Owl monkey wearing a $0.4 \mathrm{~mm}$ thick HEMA lens for 48 hours, closed eye, $\times 5700)$.

tightly packed, and the basal cells maintained a normal relation to the basement membrane.

\section{SEVERE- EPITHELIAL STRESS (THICK HYDROGEL} LENS IN CLOSED EYE)

One monkey wore a $0.4 \mathrm{~mm}$ thick lens under sutured lids on one side for 48 hours, while the opposite eye was left open. The wearing of a thick HEMA lens under closed eye conditions stripped large areas of its corneal epithelium. In other areas marked epithelial thinning was apparent (Figs. 5,6). The surviving cells were separated from each other by numerous small spaces. Pronounced cellular and nuclear deformation had occurred along with the formation of many intracellular spaces. The remaining basal cells had partially separated from the basement membrane. The areas of the cornea that had been denuded of its epithelium still possessed an intact and uninterrupted basement membrane. Structural changes in the underlying and anterior stromal tissue were not noticed.
Normal naked epithelial nerve fibres were observed in the contact lens wearing corneas except when the internal two layers of cells became severely affected by the soft lens insult (Fig. 7).

\section{Discussion}

The present study examines the short-term effect of hydrogel lens wear on a primate cornea, which closely resembles the human cornea. ${ }^{56}$ Although the experiment involved relatively few animals, the findings were consistent and therefore allowed analogies with the human situation.

The trauma from a contact lens may be due to mechanical pressure, negative suction, abrasion from lens movement, or abnormal metabolism. The present study used the same contact lens material in all fittings, and therefore the thickness variants will determine the degree of hypoxia as well as the pressure at the corneal lens interface. Disturbance of the cell organelles would be indicative of oxygen 


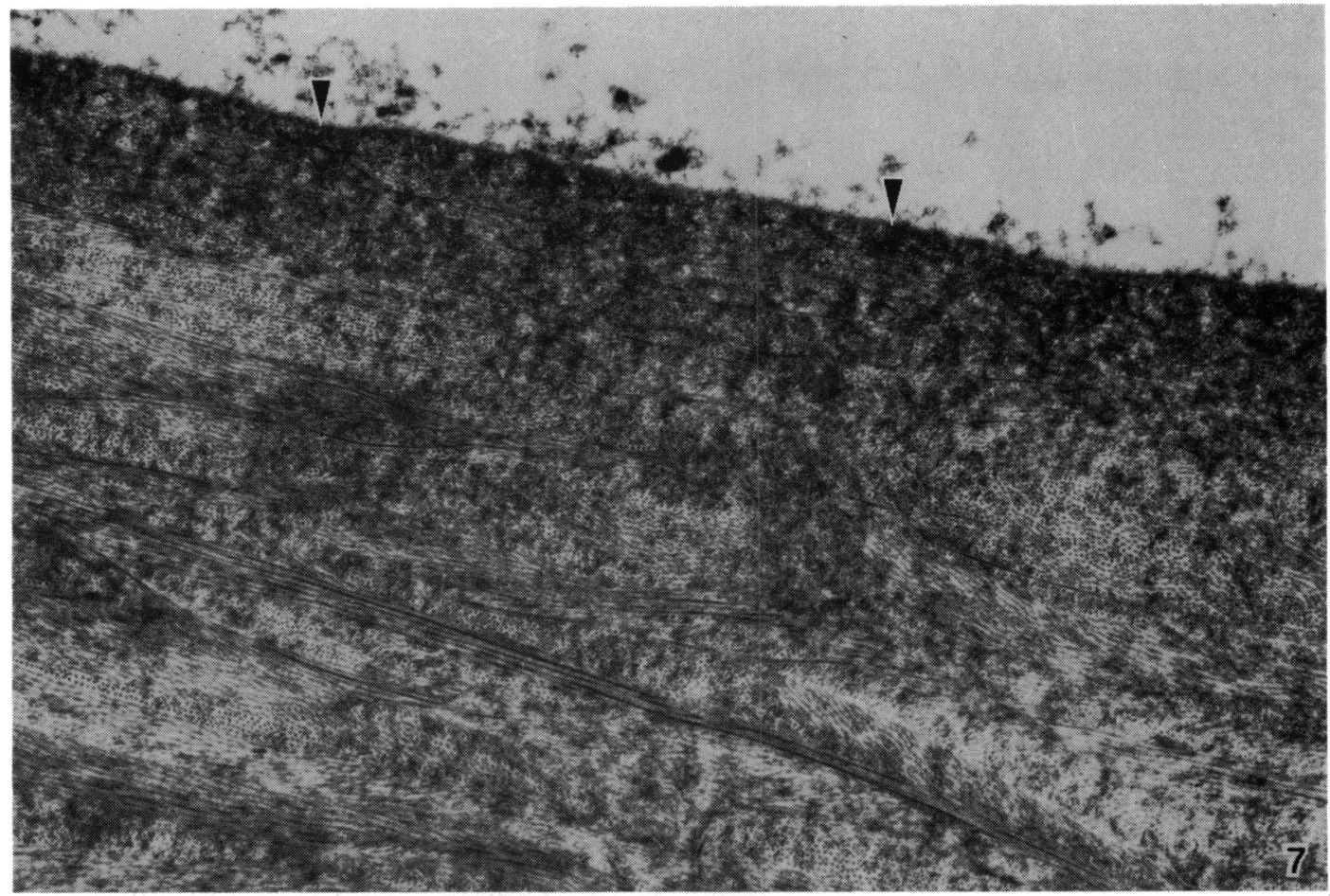

Fig. 7 Thick contact lens wearing eye with epithelial denudation. In the areas of epithelial stripping the basement membrane (triangles) remained intact. Superficial stroma appears normal. (Owl monkey wearing a $0.4 \mathrm{~mm}$ thick HEMA lens for 48 hours, closed eye, $\times 34$ 000).

deprivation, and the consequent changes could involve the water metabolism both of the epithelium and of the deeper corneal layers. On the other hand loss of surface cells, flattening of the layers, and dragging apart of the cell pattern with the development of intercellular spaces may be ascribed to direct mechanical trauma.

According to Polse and Mandell ${ }^{7}$ the minimum pressure of oxygen, below which stromal oedema occurs, is in the region 11 to $19 \mathrm{mmHg}$. These figures are a guide of the limits of normality. For a contact lens on the eye the lens $\mathrm{Dk} / \mathrm{L}$ for both the open eye state and the closed eye state can be calculated from Fatt and St Helen's figures. ${ }^{4}$ Thus the open eye lens needs a Dk/L of 5 to $10 \times 10^{-9}$ and the closed eye lens a $\mathrm{Dk} / \mathrm{L}$ of 20 to $40 \times 10^{-9}$ respectively. Table 2 shows that the thin lenses used satisfy these normality criteria for the open eye state only. The experiments with the open eye and thin lenses show, apart from a mild surface cell disturbance, no cellular abnormalities. This would confirm the criteria of Polse and Mandell. ${ }^{7}$ When the $\mathrm{Dk} / \mathrm{L}$ for the thicker lenses are considered, one must expect according to Fatt and St Helens $^{4}$ for the $0.2 \mathrm{~mm}$ thick lens a contact lens interface oxygen tension of $8 \mathrm{mmHg}$ and for the 0.4 thick lens a tension of 1 to $2 \mathrm{mmHg}$ for the open eye condition, but for the closed eye state both lenses would produce a zero oxygen tension.

The wearing of medium to thick hydrogel lenses $(0 \cdot 2-0 \cdot 4 \mathrm{~mm})$ for periods up to 15 hours with open eyes resulted in significant morphological changes in the epithelium. The epithelial thinning observed in these corneas was due to loss of cells and a flattening of the remaining ones. The cytoplasm of the surviving cells was normal except for the surface cells. The absence of epithelial oedema and the lack of abnormalities in organelles such as mitochondria suggest that anoxia has not greatly affected these cells. However, Hamano and Masaki ${ }^{8}$ have shown that mitosis decreases in the anoxic cornea, but reduced mitosis can hardly be the explanation to the loss of cells that occurred in the relatively short experimental period. The deformation of the remaining cells and sometimes also their nuclei suggests that the lens weight and lid pressure are factors in contact lens wearers, and in the absence of cytoplasmic abnormalities and oedema it is quite possible that the loss of cells is best explained by such forces. In contrast the rigid non-gas-permeable lens produced oedema and cytoplasmic changes in the epithelium. ${ }^{3}$ 
Whether the loss of epithelial cells in hard lens wearing corneas is due to trauma or anoxia may be settled only by experiments with hard gas-permeable lenses. Alternatively, it is possible that irritation induced by a rigid lens causes reflex tearing, which owing to the hypotonicity of such tears results in epithelial oedema. ${ }^{9}$ Another possible cause of epithelial oedema may be the presence of stromal oedema. Bergmanson and $\mathrm{Chu}^{3}$ reported significant stromal oedema in rigid lens wearing eyes after 24 hours, while none was seen in the open eye part of the experiments in the present study.

The thick $(0.4 \mathrm{~mm})$ HEMA lens under closed eye conditions caused large areas of epithelial stripping. Spaces were found between cells in the areas where epithelial cells were still present. Such spaces are interpreted here as indicative of oedema. The appearance of small separations between the basal cells and the basement membrane suggested that these cells were less firmly attached to the underlying cornea. Significant oedema of the stroma was noted in this cornea, and this may explain the presence of oedema among surviving epithelial cells. The basement membrane remained intact in all regions, including the areas of epithelial stripping. A similar resistance to damage of the basement membrane was also noted in the rigid lens wearing monkey. ${ }^{310}$ The regrowth of epithelial cells is greatly facilitated by the presence of an intact basement membrane, since migrating cells do not have to secrete a new membrane.

As reported previously, the epithelial nerve fibres were observed in the basal cell layer, where they remained close to the internal aspect of the cell. "In contrast to stromal fibres the epithelial axons were without Schwann cells. Unless the epithelium was severely insulted by a soft lens, its nerve fibres remained present in apparently normal numbers. Although large terminals are not a common finding in the epithelium, they were nevertheless observed in the $0.4 \mathrm{~mm}$ thick HEMA lens wearing cornea and open eye cornea. Since nerve fibres of an origin other than sensory have not been proved to be present in the primate corneal epithelium, we conclude that the observed axons are somatic sensory. The large number of mitochondria present and the absence of synaptic vesicles in these terminals support this view, though we recognise that an accumulation of mitochondria in a terminal does not allow a positive identification of origin. ${ }^{12}$ The favourable response of epithelial nerve fibres to soft lens wear correlates well with clinical experience. ${ }^{13}$ Polse $^{14}$ showed that the corneal touch threshold is not related to the availability of oxygen. It is therefore likely that the dramatic loss of sensitivity experienced with rigid
PMMA lenses is associated with trauma and oedema as well as adaptation to mechanical stimulus.

\section{CONCLUSIONS}

1. Thin $(0 \cdot 07 \mathrm{~mm})$ hydrogel $(38-43 \%$ water $)$ contact lenses worn under open eye conditions in the owl monkey caused no significant epithelial insult.

2. Medium to thick $(0 \cdot 2-0 \cdot 4 \mathrm{~mm})$ hydrogel contact lenses worn under open eye conditions resulted in a thinning of the epithelium from loss of cells and a flattening of the remaining ones. Since this thinning of the epithelium occurred without oedema, it would be difficult to discover with the slit lamp.

3 . The thick $(0.4 \mathrm{~mm})$ hydrogel contact lens under closed eye condition caused epithelial oedema and in large areas corneal denudation.

4. In none of the experiments was the basement membrane damaged. The closed eye experiment, which in places stripped the epithelium and thereby exposed the membrane, proved the durability of this structure.

5. Soft contact lens wear had no detectable effect on the epithelial innervation as long as there was not oedema.

We thank Mr Andrew Alpar for excellent photographic assistance. The lenses used in the present study were kindly provided by Bausch and Lomb, Rochester, New York and Vistaken Inc., Jacksonville, Florida.

\section{References}

1 Francois J. The rabbit corneal epithelium after wearing hard and soft contact lenses. Contact Intraoc Lens Med J 1983; 9: 267-74.

2 Ruben M. Soft contact lenses. New York: Wiley, 1978: 294-7.

3 Bergmanson JPG, Chu L W-F. Corneal response to rigid contact lens wear. Br J Ophthalmol 1982; 66: 667-75.

4 Fatt I, St Helen R. Oxygen tension under an oxygen permeable contact lens. Am J Optom Physiol Opt 1971; 49: 545-55.

5 Hogan MJ, Alvarado JA, Wedell JE. Histology of the human eye. Philadelphia, London, Toronto: Saunders, 1971: 55-111.

6 Kuwabara T. Current concepts in anatomy and histology of the cornea. Contact Intraoc Lens Med J 1978; 4: 101-32.

7 Polse KA, Mandell R. Etiology of corneal striae at hydrogel lens wear. Invest Ophthalmol Vis Sci 1976; 15: 553-6.

8 Hamano $\mathrm{H}$, Masaki $\mathrm{H}$. Effect of contact lens wear on the mitosis of corneal epithelial cells. Contact Intraoc Lens Med J 1983; 9: 133-6.

9 Schoessler JP, Lowther GE. Slit lamp observations of corneal edema. Am J Optom Physiol Opt 1971; 48: 666-71.

10 Bergmanson JPG, Chu L W-F. Contact lens induced corneal epithelial injury. Am J Optom Physiol Opt 1982; 59: 500-6.

$11 \mathrm{Lim} \mathrm{CH}$, Ruskell GL. Corneal nerve access in monkeys. Graefes Arch Klin Exp Ophthalmol 1978; 208: 15-23.

12 Bergmanson JPG. Ophthalmic terminals in the iris and the ciliary body of monkeys. Graefes Arch Klin Exp Ophthalmol 1978 206: 39-47.

13 Millodot J. Effect of the length of wear of contact lenses on corneal sensitivity. Acta Ophthalmol (Kbh) 1976; 54: 721-30.

14 Polse KA. Etiology of corncal sensitivity changes accompanying contact lens wear. Invest Ophthalmol Vis Sci 1978; 17: 1202-6. 